\title{
Synergistic effect of p-type and n-type dopants in semiconductors for efficient electrocatalytic water splitting
}

\author{
Tugce Kutlusoy ${ }^{\ddagger a}$, Spyridon Divanis ${ }^{\ddagger a}$, Riccardo Marina ${ }^{b}$, Rebecca K. Pittkowskic ${ }^{c}$ Petr \\ Krtil $^{\mathrm{c}}$ and Jan Rossmeisl ${ }^{*}$ \\ a Center of High Entropy Alloy Catalysis, Department of Chemistry, University of \\ Copenhagen, Universitetsparken 5, 2100 København $\varnothing$, Copenhagen, \\ Denmark.
}

b New Application Research, Research and Development Division, Industrie De Nora S.p.A., 20134 Milan, Italy

\begin{abstract}
c J. Heyrovsky Institute of Physical Chemistry, Academy of Sciences of the Czech Republic, Dolejskova 3, Prague 18223, Czech Republic
\end{abstract}

¥ Both authors contributed equally to this manuscript

* Corresponding author; Jan.Rossmeisl@chem.ku.dk

\begin{abstract}
The main challenge for acidic water electrolysis is the lack of active and stable oxygen evolution catalysts based on abundant materials, which are globally scalable. Iridium oxide is the only material, which is active and stable. However, Ir is extremely rare and far from scalable. There exist both active materials and stable materials, but those that are active are not stable and vice versa. In this work, we present a strategy for making stable materials active. The stable materials are semiconductors that cannot change oxidation state at relevant reaction conditions. Based on DFT calculations, we find that by adding an n-type dopant, semiconductor surfaces can bind oxygen. However, after oxygen is adsorbed, the material is again in a state where it cannot bind or desorb oxygen. By combining n-type and $\mathrm{p}$-type dopants, the reactivity can be tuned so that oxygen can be adsorbed and desorbed under reaction conditions. It turns out that the tuning can be understood from the electrostatic interactions between the dopants as well as between the dopants and the binding site. We experimentally verify that this strategy works in $\mathrm{TiO}_{2}$ by co-doping with different pairs of $n$ - and $p$-type dopants. This encourages that the co-doping approach can be used to activate stable materials, without intrinsic oxygen evolution activity, to discover new catalysts for acid water electrolysis.
\end{abstract}

\section{Introduction}

Large-scale exploration of renewable energy is vital for new sustainable energetic concepts, meeting the requirement to remove the dependence on fossil fuels. Renewable energy, based on wind or solar, is affordable but suffers from its intrinsic intermittent nature and an inconvenient regional distribution. These inherent 
disadvantages need to be mitigated by the design of novel energy storage concepts based on chemical bonds ${ }^{1}$.

Molecular hydrogen seems to be one of the primary candidates for a new energy storage system and energy vector. ${ }^{2}$ The only $\mathrm{H}_{2}$ production process fully compatible with carbon neutrality and sustainability is water electrolysis, which converts electricity into chemical energy by splitting $\mathrm{H}_{2} \mathrm{O}$ into oxygen and hydrogen. Despite significant effort devoted to optimization, water electrolysis still needs to be fundamentally improved in terms of efficiency and durability ${ }^{1,2}$.

Water electrolysis can be performed both in alkaline as well as in acid environments. ${ }^{3-}$ ${ }^{7}$ Regardless of $\mathrm{pH}$, the efficiency of the overall process is controlled by the sluggish kinetics of the oxygen evolution reaction (OER) at the anode. ${ }^{8}$

Water electrolysis in acid media would in many cases be favored over that in alkaline media, because of more facile kinetics of the cathodic hydrogen evolution, high electrolyte conductivity, and high voltage efficiencies at high current densities $^{9}$. Despite being technologically promising, the use of acid water electrolysis is limited by a lack of affordable, active, and sufficiently stable OER catalyst ${ }^{10}$.

The catalysts showing the best trade-off between durability and performance in oxygen evolution in acid media are oxides based on Ir or Ru. ${ }^{7,11}$ The low abundance and high price of these materials makes the acid water electrolysis process practically unscalable. ${ }^{12,13}$

This fact clearly stresses the importance of developing novel OER catalysts that are active, stable, and scalable for acid media oxygen evolution. The most straightforward improvement of feasibility can be achieved by an improvement of the stability while reducing or replacing the noble metal content in the catalysts. ${ }^{14,15}$

One can summarize the OER process as a sequence of four consecutive oneelectron/proton transfer steps, which require the formation of three surface confined intermediates ${ }^{16,17}$. The individual reaction steps are:

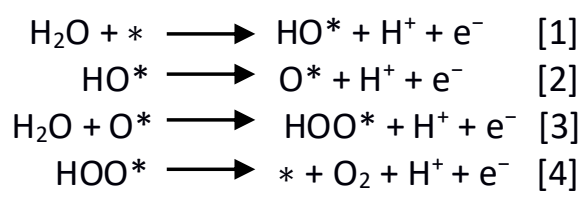

In an ideal catalyst all reaction intermediates, $\mathrm{HO}^{*}, \mathrm{O}^{*}$, and, $\mathrm{HOO}^{*}$, bind on the surface in such a way that all reaction steps [1]-[4] take place at the equilibrium potential of $1.23 \mathrm{~V}$ (i.e., each reaction step is driven by the energy of $1.23 \mathrm{eV})$.

The main factor limiting the process optimization of water oxidation is the scaling relation between the first $\left(\mathrm{HO}^{*}\right)$ and the third intermediate $\left(\mathrm{HOO}^{*}\right) \cdot{ }^{18-20}$ As both intermediates bind identically to the surface, a constant difference in adsorption energies of $3.2 \mathrm{eV}$ is obtained, regardless of catalyst material. This situation greatly deviates from the ideal case where the difference in $\mathrm{HO}^{*}$ and $\mathrm{HOO}^{*}$ adsorption energies should equal $2.46 \mathrm{eV} .{ }^{18,19}$ The validity of the scaling relation has been shown also experimentally. ${ }^{21,22}$

To avoid the feasibility restrictions of the state-of-the-art OER catalysts based on Ir and $\mathrm{Ru}$ discussed above, we propose an alternative approach: activating 
semiconducting materials like, e.g., $\mathrm{TiO}_{2}$, to form potent $\mathrm{OER}$ catalysts of high stability. The stability of many semiconducting oxides is inherently related to their wide bandgap, which, in turn, leads to low catalytic activity. The poor performance of $\mathrm{TiO}_{2}$, chosen as a model semiconductor, in electrochemical water oxidation is well established. ${ }^{23-28}$ Doping $\mathrm{TiO}_{2}$ to increase its OER activity is also well established. ${ }^{27-32}$ This work demonstrates the activation of $\mathrm{TiO}_{2}$ for OER catalysis by simultaneous $\mathrm{p}$ type and n-type doping (further denoted as "co-doping"). While semiconductor codoping is commonly used to tune the bandgap width in the field of photocatalysis ${ }^{33-}$ 35 , such co-doping has never been - to the best of our knowledge - applied in electrocatalysis. Here, we present a theoretical approach outlining a systematic way to improve the catalytic activity of semiconductors through a co-doping approach. The co-doping approach minimizes the trade-off between stability and activity by preserving the intrinsic stability of the semiconducting material while increasing its activity because of the electronic states created by the dopants. The theoretical predictions are experimentally verified on model catalysts based on co-doped $\mathrm{TiO}_{2}$.

\section{Results and discussion}

\section{Co-doping approach}

For semiconducting oxides, any computational approach needs to avoid finite-size effects leading to computational artefacts ${ }^{19}$. With the elimination of the finite size effect, it is possible to computationally assess activity trends of heavily doped semiconductors, e.g. $\mathrm{TiO}_{2}$, and position these materials on the theoretical OER activity volcano to draw a comparison with other materials. The mechanism of $\mathrm{TiO}_{2}$ activation via co-doping is shown on the rutile polymorph to maintain structural similarity with the state-of-the-art OER catalysts in acid media $\left(\mathrm{RuO}_{2}, \mathrm{IrO}_{2}\right)$.

The poor conductivity of $\mathrm{TiO}_{2}$ can be improved by $\mathrm{n}$-type doping (i.e., by adding donors of electrons), which can directly provide electrons to the conduction band $(C B)$ or create states in the bandgap close to the $C B$ edge. The effect of the n-type dopants can be demonstrated on a model system, where $\mathrm{TiO}_{2}$ is doped with $\mathrm{V}$. Extending the analysis to $\mathrm{p}$-type doped $\mathrm{TiO}_{2}$ (i.e., introducing acceptors of electrons e.g., Rh) one finds a different type of behavior. P-type dopants increase the probability of forming a hole in the valence band (VB). In other words, p-type dopants deplete the electron density of the VB forcing the Fermi level to move close to the electronic states created by the $\mathrm{p}$-type dopants. The effect of dopants on the $\mathrm{TiO}_{2}$ electronic structure is depicted in Fig. 1, which shows the projected density of states (pDOS) of the $\mathrm{TiO}_{2}$ rutile structure doped with $n$-type (V) and p-type dopant (Rh), as well as the co-doped structure $(\mathrm{V}+\mathrm{Rh})$. Both doping and co-doping the $\mathrm{TiO}_{2}$ with $\mathrm{Rh}$ and $\mathrm{V}$ create states in the bandgap, which due to their occupancy may interact with the reagent water molecules. These newly created states may eventually (in the case of co-doping) form a new, rather narrow, electronic band that crosses the Fermi level. In this way, the electronic structure of the co-doped $\mathrm{TiO}_{2}$ resembles that of a conductor. The behavior of the Fermi level, which varies according to the type of dopant introduced, is in accordance with literature ${ }^{36}$. 

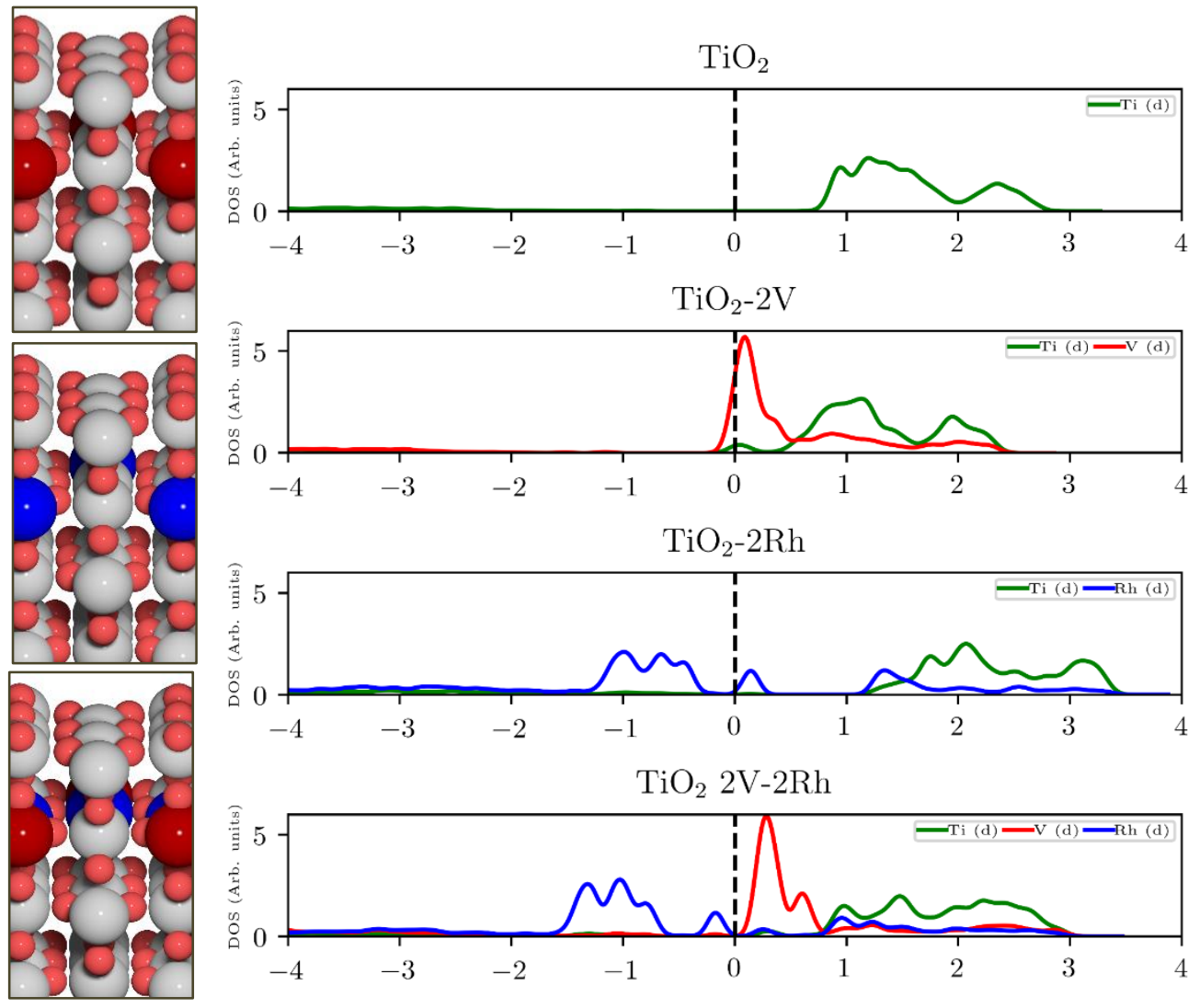

$\mathrm{TiO}_{2}-2 \mathrm{~V}$

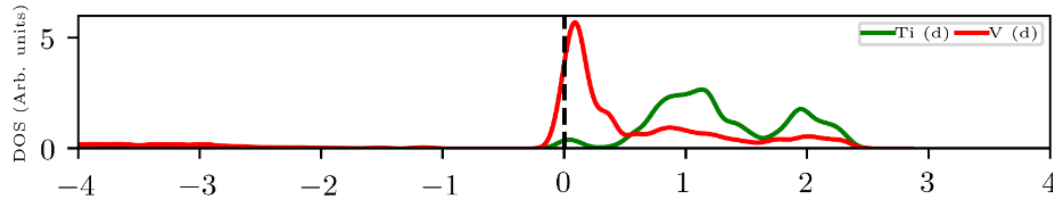

$\mathrm{TiO}_{2}-2 \mathrm{Rh}$

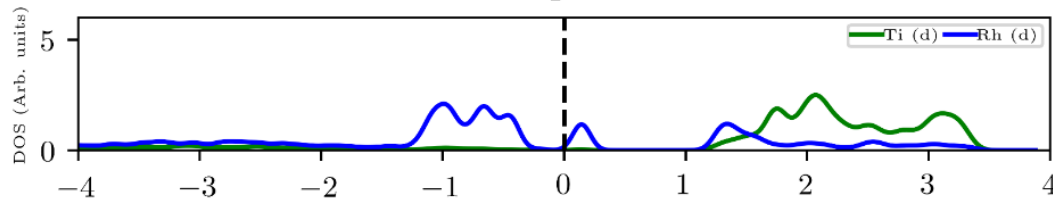

$\mathrm{TiO}_{2} 2 \mathrm{~V}-2 \mathrm{Rh}$

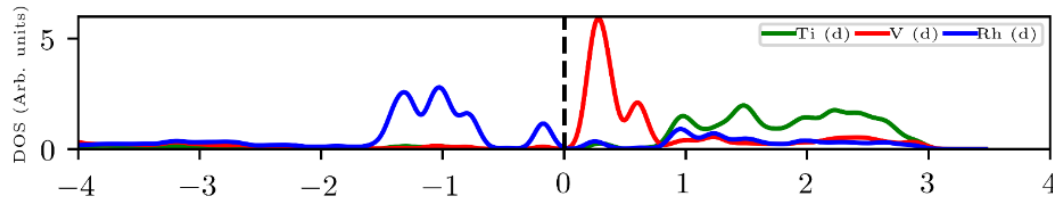

Figure 1) Projected density of states (pDOS) of pristine and doped $\mathrm{TiO}_{2}$ and corresponding atomic structures of rutile-type $\mathrm{TiO}_{2}$ (110) with $\mathrm{V}(\mathrm{red}), \mathrm{Rh}$ (blue) (from top to bottom: $n$-doped, p-doped, co-doped). pDOS From top to bottom: pristine $\mathrm{TiO}_{2} ; \mathrm{V}$-doped $\mathrm{TiO}_{2}$ : the $V$ d-states intersect with the Fermi level. The majority of the states is located at energies above the Fermi level; Rh-doped $\mathrm{TiO}_{2}$ : The $\mathrm{Rh}$ d-states are located mostly around the Fermi level and also at higher energies above the Fermi level; and $\mathrm{V}$-Rh co-doped $\mathrm{TiO}_{2}$. The dopants' $d$-states populate the bandgap and are placed around the Fermi level.

a)

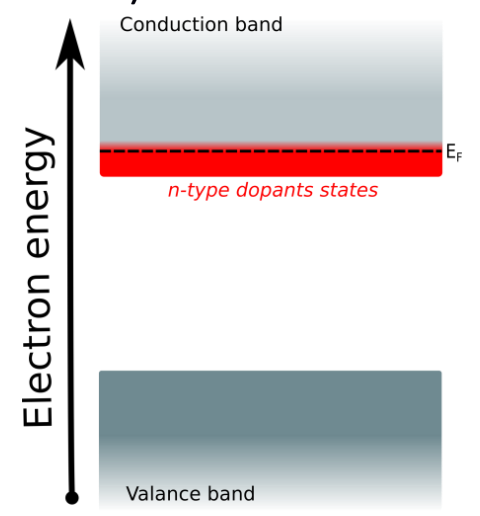

b)
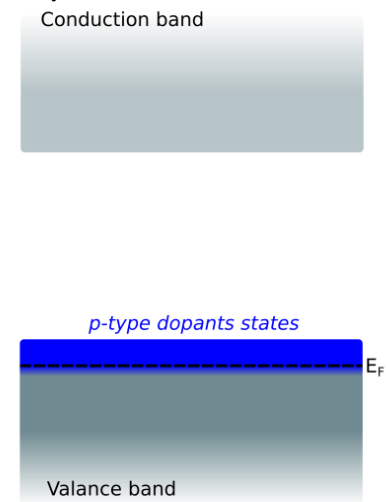

c)

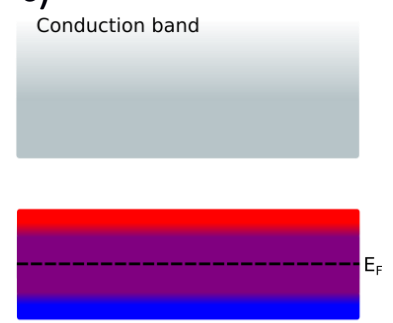

co-doped states

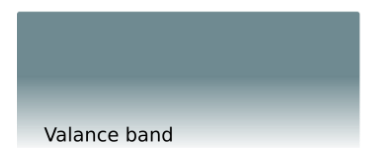

Figure 2) a) Insertion of an n-type dopant in a semiconductor, b) Insertion of a p-type dopant in a semiconductor, c) Simultaneous Insertion of an $n$-type and a $p$-type dopant in a semiconductor and the creation of the compensated electron states. 
Creating these pseudo-conductor states in the bandgap of a stable, essentially semiconducting, material such as $\mathrm{TiO}_{2}$ has a fundamental impact on the catalytic behavior of these materials. The doping/ co-doping approach allows tuning the reactivity of the surface. Most $n$-type dopants can easily provide an electron to the binding site. Combining $n$ - and $\mathrm{p}$-type dopants, it becomes increasingly difficult for electrons to leave the dopant complex and participate in the surface binding. Therefore, the co-doping strategy may be able to make stable semiconducting oxides active for OER catalysis (see Fig. 2).

The OER free energy diagrams presented in Fig. 3a identify the oxidation of $\mathrm{HO}^{*}$ to $\mathrm{O}^{*}$ as the potential determining step (PDS) for pristine $\mathrm{TiO}_{2}$ (eq. [2]). This places the rutile polymorph of $\mathrm{TiO}_{2}$ on the weak binding branch of the OER activity volcano (see Fig. $3 b$, grey). The n-type dopants change the PDS from the $\mathrm{HO}^{*} \rightarrow \mathrm{O}^{*}$ oxidation to $\mathrm{O}^{*} \rightarrow$ HOO*. This shifts the $n$-doped $\mathrm{TiO}_{2}$ from the weak binding branch to the strong binding branch of the activity volcano (Fig. $\mathbf{3} \boldsymbol{b}$, red data point). Hence, $\mathrm{n}$-type doping makes the rutile structure highly reactive resulting in too strong oxygen adsorption. This causes an accumulation of chemisorbed oxygen intermediates eventually blocking the surface. After blocking the surface, the activity of the n-type doped rutile does not differ from that of pristine $\mathrm{TiO}_{2}$ (see Fig. 2b).

DFT analysis of the effect of $p$-type dopants on the theoretically assessed OER activity places the $\mathrm{p}$-doped $\mathrm{TiO}_{2}$ on the weak binding side of the volcano and the predicted activity shows only a marginal improvement over that of pristine $\mathrm{TiO}_{2}$. Therefore, in conclusion, the doping of $\mathrm{TiO}_{2}$ with a single type of dopant does not improve its OER activity since (i) the n-type doping causes primarily an accumulation of strongly chemisorbed oxygen on the surface and (ii) the activity of the p-type doped materials does not significantly change the activity of the original material.

After examining the effect of both p-type and n-type doping on the OER activity of the $\mathrm{TiO}_{2}$ surface, one may naturally consider simultaneous co-doping of $n$ - and $p$-type elements. The OER-related catalytic activity of the co-doped $\mathrm{TiO}_{2}$ is represented in Fig. 3 by the purple-colored data. The theoretical activity of co-doped $\mathrm{TiO}_{2}$ significantly differs from those of pristine as well as $\mathrm{n}$ - and $\mathrm{p}$-type doped $\mathrm{TiO}_{2}$. The theoretical prediction places the co-doped $\mathrm{TiO}_{2}$ on the weak branch of the volcano; however, the theoretical overpotential is greatly reduced, mainly due to an optimized binding of the O* intermediate (Fig. 3a). 
a)

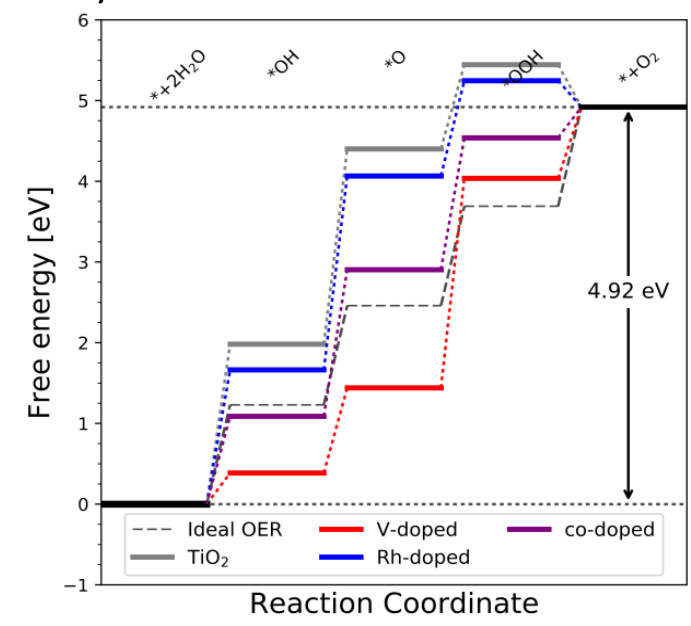

b)

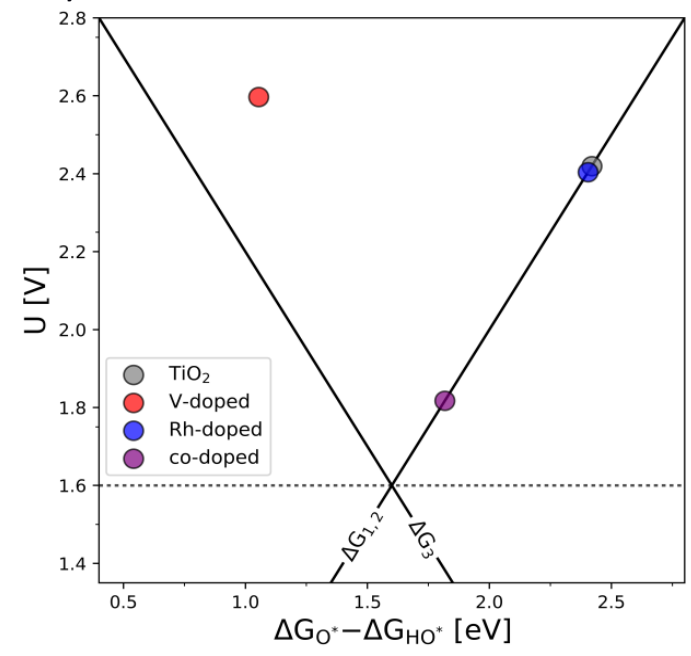

Figure 3 a) Free energy diagram of the pure (grey), single doped (blue - Rh and red - V), and co-doped (purple) $\mathrm{TiO}_{2}$ and b) the corresponding activity volcano depicting the increased catalytic performance of the co-doped structure.

\section{Coulombic effect}

The improved binding of OER intermediates at the co-doped $\mathrm{TiO}_{2}$ surface retains a local character that is dependent on the relative position of the dopants in the structure. To investigate this effect, we performed a series of calculations for different local arrangements of both dopants in the unit cell by varying the relative distance of the dopants as well as their distance to the binding site. Based on the relative position of both Rh and $V$, the resulting catalysts may reside on either branch of the theoretical volcano (see Supporting Information, Fig. S1).

To further outline this phenomenon, we conducted calculations with one $V$ atom in the $\mathrm{TiO}_{2}$ structure with a gradually increasing distance between the dopant and the adsorbed intermediate. We selected $\mathrm{HO}^{*}$ as the model intermediate since it is bound on the surface via a single bond. The use of a single dopant does not introduce finitesize effects. The corresponding atomic structure representations are given in the SI, Fig. S2. By varying the distance of dopants to the adsorbed intermediate, the binding energy of the intermediate is changed, which has previously been shown for $\mathrm{TiO}_{2}$ doped with molybdenum. ${ }^{37}$ In particular, we find that a linear correlation exists between the $\mathrm{HO}^{*}$ binding energy and the reciprocal distance between the dopant and the binding site. The $\mathrm{HO}^{*}$ binding energy shows the same variation with the reciprocal distance of the vanadium atom and the binding titanium atom, as if $\mathrm{V}$ delivers an electron to HO*, see Fig $\mathbf{4 a}$. The generality of the Coulombic effect between the dopant and the binding intermediate can be shown by extending the analysis to $\mathrm{SrTiO}_{3}$, see Supporting Information Fig. S3. Here, the same dependence of the HO* binding energy on the reciprocal distance $1 / \mathrm{r}$ is obtained. The interaction between $\mathrm{V}^{+}$and $\mathrm{HO}^{-}$ is thus a simple Coulombic term; the closer the dopant is to the binding site the 
stronger the binding due to the attraction between the negative and positive charges. This Coulombic effect between the dopant and the binding intermediate as a function of dopant position in the structure is schematically represented in Fig. $\mathbf{4 b}$.

a)

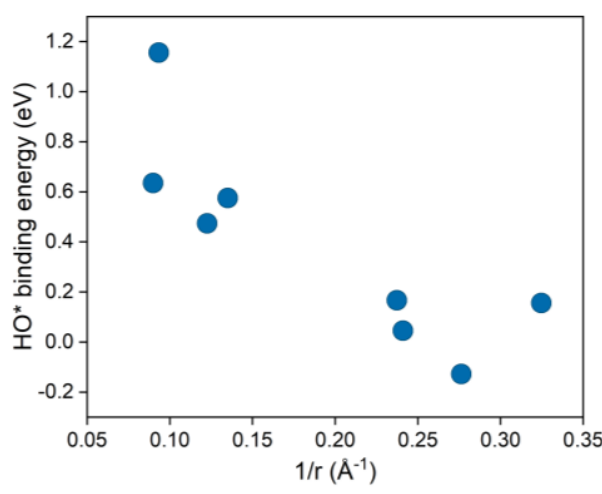

b)

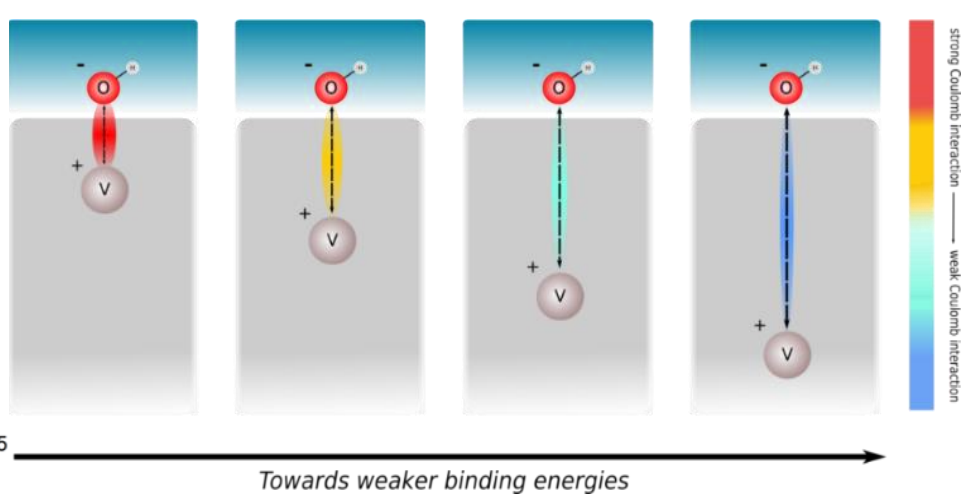

Figure 4 a) Binding energy of $\mathrm{HO}^{*}$ against $1 / r$, where $r$ is the distance of the dopant $(V)$ from the intermediate in a $\mathrm{TiO}_{2}$ structure for varies placements in the structure (cus and bridge sites, the corresponding structures are given in SI, Fig.S2) b) Schematic representation of the effect of the distance between the intermediate $\left(\mathrm{HO}^{*}\right)$ and the dopant $(\mathrm{V})$ on the binding energy of the intermediate

Extending this approach to the co-doped case, the reactivity of the binding site will, therefore, depend on the distance to the dopants, the relative distance of dopants, and the dielectric constant of the semiconductor. It is more complicated to show the Coulombic interaction directly for the co-doped case, as it will depend on the partial charge and on the dopants' and adsorbate positions, respectively; however, we see no reason why this picture should not apply for many dopants also. This does not mean that the nature of the dopant is not important for the binding energy, as this determines the position of the states in the bandgap. It means, however, that for a given set of dopants, semiconductors, and intermediates the variations in binding energies are governed by electrostatics. A more detailed discussion of the Coulombic effect further showing the generality of the concept irrespective of the dopant is given in the Supporting Information, see Fig. S1-S10 for both doped and co-doped $\mathrm{TiO}_{2}$.

The generality of the co-doping concept can further be demonstrated by extending it to another semiconducting system of significant stability - $\mathrm{SrTiO}_{3}$ perovskite. The chosen perovskite structure shows (in its non-doped state) a band structure similar to that of $\mathrm{TiO}_{2}$. It also shows outstanding chemical stability but its OER-related electrocatalytic activity is rather low, in part also due to its wide bandgap. ${ }^{38,39}$ As in the case of $\mathrm{TiO}_{2}$, $\mathrm{Rh}$ and $\mathrm{V}$ were used as the p-type and n-type dopant, respectively. The results of the thermodynamic analysis of the OER catalysis on pristine and doped $\mathrm{SrTiO}_{3}$ structures are summarized in Fig. 5. 
a)

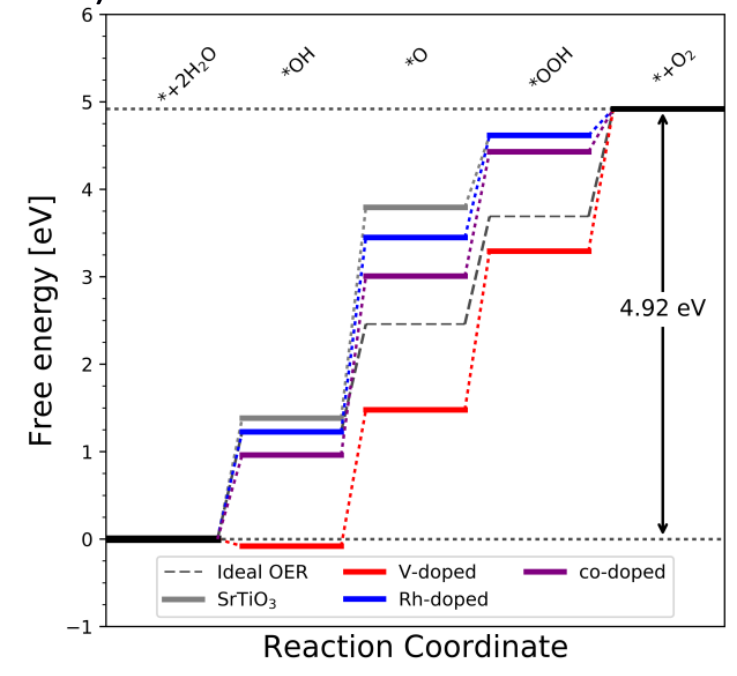

b)

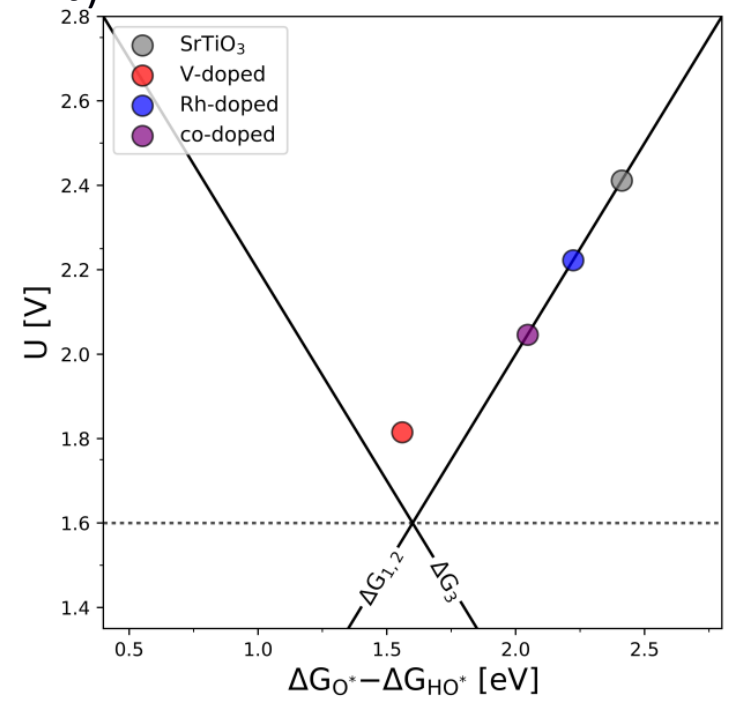

Figure $\mathbf{5}$ a) Free energy diagram of the pure (grey), doped (blue and red), and co-doped (purple) $\mathrm{SrTiO}_{3}$. As in the case of $\mathrm{TiO}_{2}$, the energy level of the intermediates corresponding to the co-doped structure are in between the $p$-and $n$-states $b)$ the corresponding activity volcano.

The results presented in Fig. 5 essentially reproduce the trends predicted for the doped and co-doped $\mathrm{TiO}_{2}$. The only difference in the behavior of $\mathrm{SrTiO}_{3}$ is that $n$-type doping alone yields a better OER activity than the co-doped $\mathrm{SrTiO}_{3}$. This observation shows that the partially oxidized $\mathrm{SrTiO}_{3}$ corresponds to the most stable phase in the overall OER reaction sequence. The co-doped structure lies energetically in between the $\mathrm{p}$ - and the n-type doped $\mathrm{SrTiO}_{3}$ as in the case of $\mathrm{TiO}_{2}$ (Fig. 5). The theoretical activity of the co-doped $\mathrm{SrTiO}_{3}$ is much lower than that of co-doped $\mathrm{TiO}_{2}$. This unfavorable trend can, however, be reverted by partial substitution of $\mathrm{Sr}$ with $\mathrm{Ba}$ in the A-site of the perovskite structure, which activates the co-doped perovskite in the OER process (Fig. 6). Schematic representation of the co-doped perovskite structures $\mathrm{SrTiO}_{3}$ and $\mathrm{Ba}_{0.16} \mathrm{Sr}_{0.84} \mathrm{TiO}_{3}$ are shown in the SI, Fig. S11. 


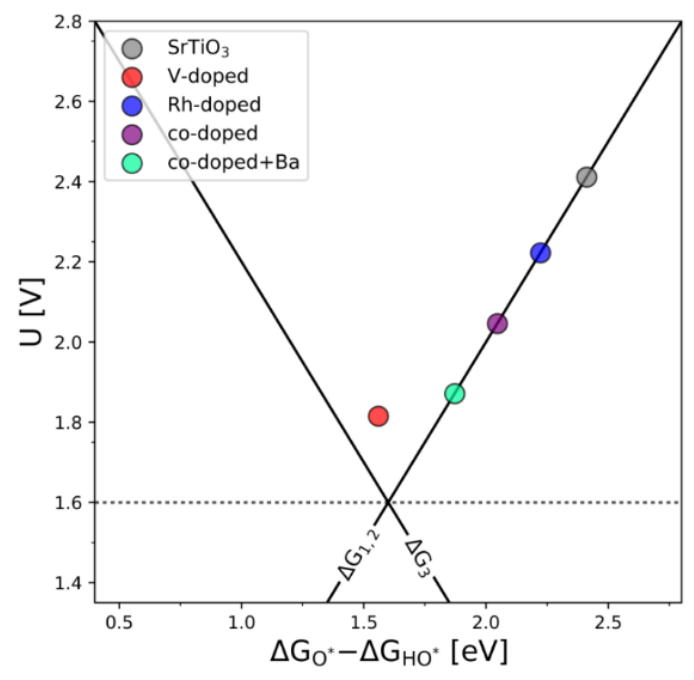

Figure 6. The OER activity Volcano of V-doped, Rh-doped, and V-Rh co-doped $\mathrm{SrTiO}_{3}$ with the addition of the $\mathrm{V}$-Rh co-doped $\mathrm{Sr}_{0.84} \mathrm{Ba}_{0.16} \mathrm{TiO}_{3}$ structure, which resembles the co-doped $\mathrm{TiO}_{2}$ in its activity.

The improvement of the OER activity of perovskite-based catalysts connected with control of the redox state of the B-site cation in the perovskite structure has previously been reported ${ }^{3}$. This, however, cannot explain the observed effect of increased activity due to Ba substitution here, since the Ba s-valence orbitals are not catalytically active. A possible explanation could be that alterations in the overall band structure occur due to the Ba s-orbitals pushing the electronic states of both dopants to a more favorable position to facilitate the adsorption of OER intermediates.

To further test the generality of the co-doping concept, we have broadened the initial screening of $n$ - and p-type dopants of $\mathrm{TiO}_{2}$ and $\mathrm{SrTiO}_{3}$ to include $\mathrm{V}, \mathrm{Nb}, \mathrm{Ta}, \mathrm{Mo}$, and $\mathrm{W}$, as n-type dopants, and $\mathrm{Rh}, \mathrm{Mn}, \mathrm{Pd}$, and Ru as p-type dopants. Extending the co-doping hypothesis, we have chosen the following additional pairs of $p$ and $n$-type dopants: All n-type dopants ( $, \mathrm{Nb}, \mathrm{Ta}, \mathrm{Mo}, \mathrm{W}$ ) are paired with $\mathrm{Rh}, \mathrm{Mn}, \mathrm{Pd}, \mathrm{Ru}$. All combinations of $\mathrm{p}$ - and n-type dopants lead to electronic structures, which in their OER activity surpass the OER activity of pristine $\mathrm{TiO}_{2}$. (See Fig. S12 in the Supporting Information).

It is necessary to point out that the calculated bandgap energies of $\mathrm{TiO}_{2}$ and $\mathrm{SrTiO}_{3}$ are severely underestimated compared to experimental values, which is a well-known problem of DFT ${ }^{40}$. This may directly affect the calculated binding energies of the OER intermediates. U-correction partially compensates for the deviation of calculations from experiments, ${ }^{36,41}$ but this approach also has its drawbacks. The U-value used for each dopant is different, thus comparing the activity of different systems is not straightforward. One also needs to reflect that the binding energy of the intermediates is scaling with the value of U-correction. The details of U-corrected bandgap energies are given in the SI, Fig. S13 - Fig. S15. The absolute binding energies 
may be affected by systematic errors in the simulations and the inherent uncertainty of the position of individual combinations of $n$ - and $p$-type dopants. However, the effect of the co-doping will apply even if the absolute values change. Hence, the computational screening approach presented here allows narrowing the selection of prospective dopants.

An ultimate step in the co-doping optimization of OER activity of semiconductors can be seen in further doping of the already co-doped structures $\mathrm{Ti}(\mathrm{RhV}) \mathrm{O}_{2}$ and $\mathrm{Ba}_{0.16} \mathrm{Sr}_{0.84} \mathrm{Ti}(\mathrm{RhV}) \mathrm{O}_{3}$, with both $\mathrm{n}$-type and p-type dopants The results of this computational approach are summarized in Fig. 7, showing the position of these materials on the theoretical volcano. For both rutile and perovskite structures, introducing several extra dopants can further increase the OER activity of the codoped structures. This can be traced back to variations in the band structure as a result of adding the extra dopant. The Rh and V co-doping states dominate however the behavior, which places all structures on the weak binding side of the OER volcano.

a)

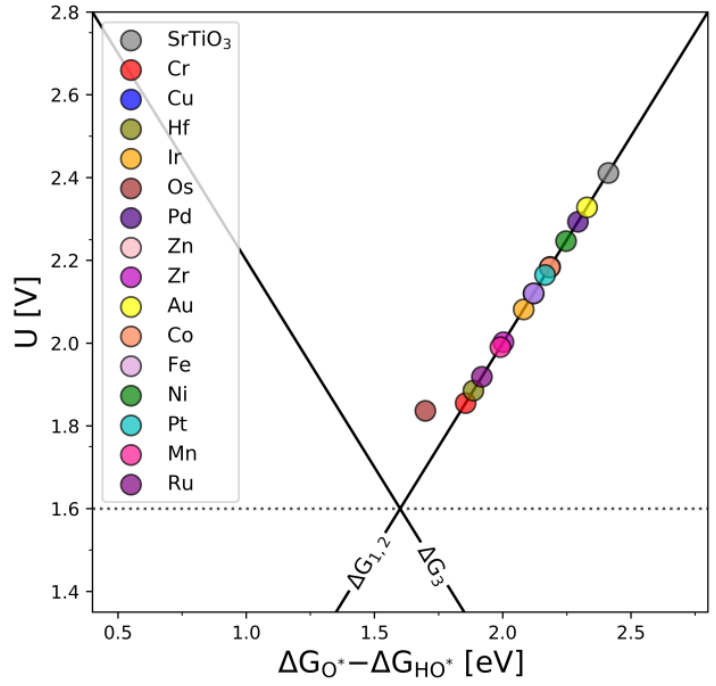

b)

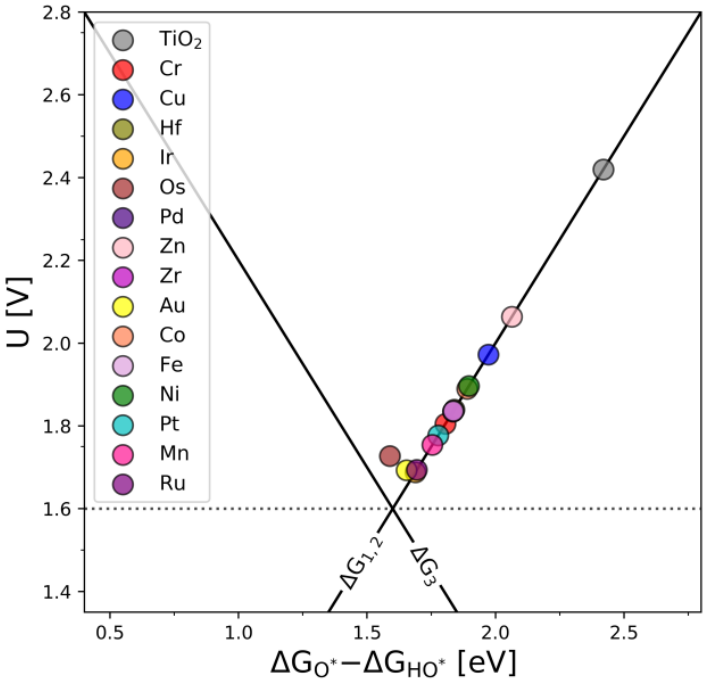

Figure 7. OER activity volcanoes for $\mathrm{Sr}_{0.16} \mathrm{Ba}_{0.84} \mathrm{Ti}(\mathrm{RhV}) \mathrm{O}_{3}(\boldsymbol{a})$ and $\mathrm{Ti}(\mathrm{RhV}) \mathrm{O}_{2}(\boldsymbol{b})$ doped with extra transition metal dopants (additional d-states).

It may be concluded that the activity improvement reflected in Fig. 7 results from the joint effect of the compensated states and the additional $d$-states provided by the extra dopant to the active part of the system's band structure. A representative example of the pDOS for such a system is shown in the Supporting Information, Fig. S16, for the case of $\mathrm{Mn}, \mathrm{V}$, and Fe dopants introduced in the $\mathrm{TiO}_{2}$ structure.

While the theoretical analysis suggests that co-doping $\mathrm{TiO}_{2}$ has the potential to disrupt the OER catalyst design, the viability of the concept needs to be experimentally 
verified. While the theoretical screening of prospective co-doped systems is relatively affordable, actual synthesis of the theoretically predicted systems is less straightforward. To prove the theoretical concept, one needs to select a system reflecting the main features of the theoretical concept while maintaining sufficient synthetic facility. It needs to be stressed that theoretically conceived materials represent metastable phases and, thus, the experimental proof of concept must rely on a low-temperature synthetic approach. ${ }^{42,43}$ This favors $\mathrm{TiO}_{2}$-based systems over $\mathrm{SrTiO}_{3}$-based materials. ${ }^{44}$

Experimental confirmation of the predicted superior OER activity of the co-doped $\mathrm{TiO}_{2}$ materials is still relatively complicated. It needs to be noted that rutile, which is the basis of the theoretical analysis (it represents the thermodynamically stable titania polymorph), is difficult to prepare at low temperatures. The absence of convenient low-temperature synthesis also complicates the possible stabilization of large amounts of doping cations in its structure, since the theoretically predicted chemical compositions are likely to remain stable if the thermal treatment does not exceed 500 ${ }^{\circ} \mathrm{C}$. These synthetic approaches, however, yield the thermodynamically metastable anatase titania polymorph. ${ }^{44,45}$ This fact should not represent a fundamental problem since anatase is also an n-type semiconductor and features a similar bandgap and similar band edge energies as the rutile polymorph. ${ }^{46,47}$

Single-doped and co-doped $\mathrm{TiO}_{2}$ nanoparticles were prepared using the spray-freeze freeze-dry approach ${ }^{48,49}$. The doping levels achieved in all prepared $\mathrm{TiO}_{2}$ materials were equal to a substitution of $20 \%$ of cationic positions, either with one dopant $(\mathrm{Cr}$ or $\mathrm{Mn}$ ) or two dopants (yielding both $\mathrm{n}$ - and $\mathrm{p}$-type doping) in equal amounts. All synthesized titanium oxides materials were nanocrystalline and conform to the anatase structure (see Fig. 8a). Doping the anatase structure did not cause any significant variation in the lattice parameters (obtained by Rietveld refinement), the characteristic particle size, and the coherent domain sizes. Details of the structural characterization of the obtained materials are given in the Supporting Information (see Fig.S17-18 and Table S1). 
Although the XRD-based structure of the prepared materials conforms to that of the anatase, the same cannot be said of their electronic structure as can be inferred from measured UV-Vis spectra (see Fig. $8 \boldsymbol{b}$ ). The UV-Vis spectrum of pure anatase is typical for a wide bandgap semiconductor characterized with a clearly defined band gap of more than $3 \mathrm{eV}$. This type of behavior is not replicated in the doped or co-doped materials. The pronounced onset of the absorption in the visible region is already evident in the materials doped with a single dopant ( $\mathrm{Mn}, \mathrm{Cr}$ in Fig. $\mathbf{8 b}$ ). For materials subject to simultaneous $\mathrm{n}$ - and $\mathrm{p}$-type doping, one observes a continuous absorption extending over a rather broad interval of energies, spanning for c.a. 2.0 to $2.5 \mathrm{eV}$. This broad absorption band is further complemented by a resolved localized absorption band located at ca. $1 \mathrm{eV}$, characteristic namely for materials doped with $\mathrm{Co}$. This type of behavior is generally compatible with the conductivity behavior predicted in the DFT calculations. It needs to be noted that the co-doped $\mathrm{TiO}_{2}$ materials do not retain the behavior characteristic of the pure $\mathrm{TiO}_{2}$, namely, they do not show characteristic photo-electrochemical activity.

a)

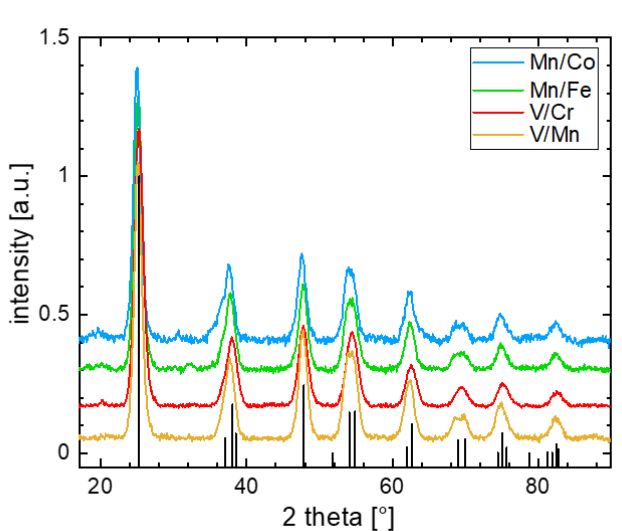

b)

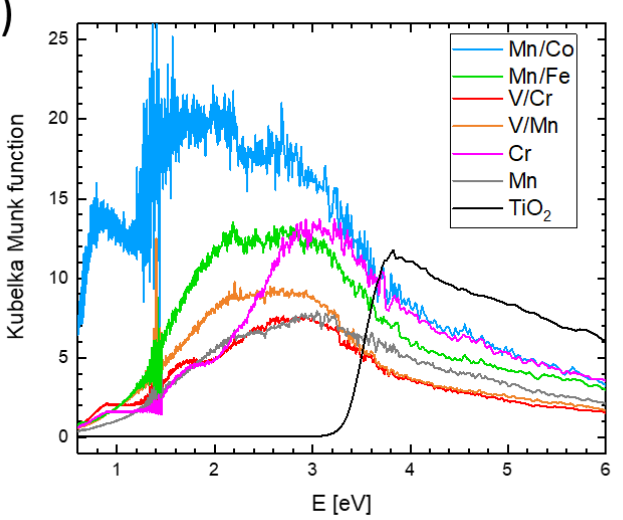

Figure 8. a) X-ray diffraction patterns of the synthesized $\mathrm{n}$ and $\mathrm{p}$ co-doped $\mathrm{TiO}_{2}$ nanoparticles, the reference pattern for $\mathrm{TiO}_{2}$ in anatase structure is included as the black line pattern and $\boldsymbol{b}$ ) $\mathrm{UV}$-vis spectra of all synthesized doped $\mathrm{TiO}_{2}$ nanoparticles including the pristine $\mathrm{TiO}_{2}$ anatase reference. 
The OER activity of the doped $\mathrm{TiO}_{2}$ materials was assessed in acid media and compared with that of the benchmark $\mathrm{TiO}_{2}$ sample (see Fig. 9) ${ }^{50}$. The linear sweep voltammograms of the co-doped $\mathrm{TiO}_{2}$ samples are presented in Fig. 9a. For better comparison, the potential necessary to drive a current density of $50 \mu \mathrm{A} / \mathrm{cm}^{2}$ is taken as an estimate of the oxygen evolution activity (Fig. 9 b). The estimated overpotentials show that the behavior of materials doped with a single cation and that of the $n$ - and $p$ - co-doped materials significantly differs, despite the similarity in UV-Vis spectra. While the co-doped materials clearly outperform the pure $\mathrm{TiO}_{2}$ benchmark sample, the activity of the materials doped with a single cation remains comparable with that of the benchmark, i.e., non-doped $\mathrm{TiO}_{2}$. It can be concluded that only co-doping has a pronounced effect on the OER activity of the $\mathrm{TiO}_{2}$ electrodes (see Fig. 9b). Although the observed behavior is essentially in line with the DFT-based theoretical prediction presented above, the actual OER activity seems to also be affected by the chemical nature of the dopants.
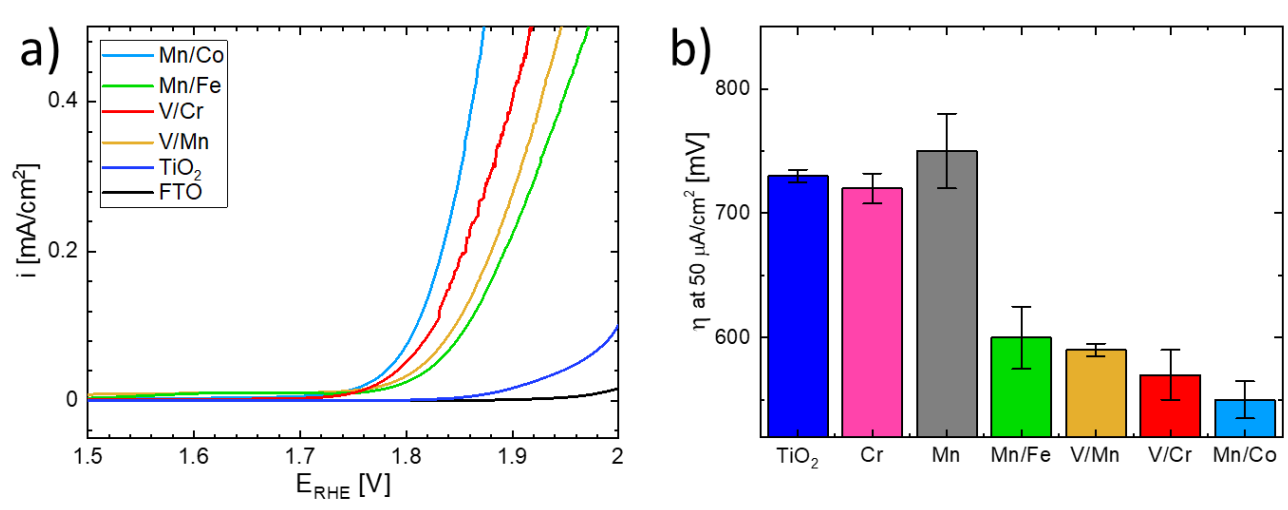

Figure 9. a) linear sweep voltammograms of the synthesized co-doped $\mathrm{TiO}_{2}$ nanoparticles and the $\mathrm{TiO}_{2}$ benchmark material. Voltammograms were recorded in $0.1 \mathrm{M} \mathrm{HClO}_{4}$ using FTO as a current collector and b) overpotential $\eta$ necessary to drive a current density of $50 \mu \mathrm{A} / \mathrm{cm}^{2}$ on the co-doped $\mathrm{TiO}_{2}$ nanoparticles, single dopant $\mathrm{TiO}_{2}$ nanoparticles, and the $\mathrm{TiO}_{2}$ benchmark sample. The values included are the mean averages of three individual measurements. The assignment of individual samples is given in the figure legend.

The $\mathrm{Mn} / \mathrm{Co}$ co-doped structure exhibits the highest activity (i.e., the lowest overpotential) among all the co-doped structures as shown in Fig. $\mathbf{9 b}$. One could argue that since $\mathrm{Mn}$ and Co-based oxides themselves are relatively good catalysts for OER ${ }^{51-}$ 55 , their phase separation may be responsible for the increased activity and not the compensated states of the dopants. On the other hand, the second-best performance is exhibited by the $\mathrm{V} / \mathrm{Cr}$ co-doped structure and since $\mathrm{V}$ and $\mathrm{Cr}$ do not form good OER catalysts, we can robustly argue that the compensation effect is responsible for their increased catalytic activity. It has to be noted, however, that the stability of the prepared doped and co-doped $\mathrm{TiO}_{2}$ nanoparticles is limited during oxygen evolution in acid media, which can be explained by the random distribution of dopants in the particles. Dopants placed on the particle surface lead to leaching and loss of the 
dopants which in turn decreases the OER activity. Choosing a synthetic strategy that confines the dopants to the bulk ${ }^{38}$ as an alternative could be an approach to increase the stability of these materials.

\section{Conclusions}

Simultaneous doping of wide bandgap semiconductors with both $\mathrm{n}$ - and $\mathrm{p}$-type dopants affects both the electronic structure as well as the catalytic behavior of semiconducting oxides. This co-doping significantly improves the OER activity of these materials. $\mathrm{TiO}_{2}$ and $\mathrm{SrTiO}_{3}$, known for their stability under acidic and alkaline conditions and their poor performance in OER catalysis, were chosen as model systems to outline the co-doping effect computationally. The inclusion of both n-type and $p$-type dopants in these structures causes a compensation effect between the electronic states of the dopants, resulting in an alteration of the band structure, which consequently lowers the overpotential of OER. According to the pDOS, the dopants form a new band that aligns the Fermi level at an energetic position favorable for water oxidation. The calculations further reveal a sensitivity of the binding energies of the intermediates to the relative position of the dopants. Combining this local structure sensitivity with the Sabatier principle can be used to optimize the chemical composition of the co-doped catalysts. The theoretical predictions were confirmed experimentally on the doped and co-doped $\mathrm{TiO}_{2}$ - anatase. The formation of the pseudo-conductor band is reflected in the significant coloration of the doped anatase. While a similar change of the electronic structure can be seen for a single-doped system, only $\mathrm{n}$ - and $\mathrm{p}$-type co-doped materials show an actual significant improvement of the catalytic activity in oxygen-evolving reactions.

\section{Computational Methods}

The Grid-based Projector Augmented Wave (GPAW) ${ }^{56,57}$ package along with the Atomic Simulation Environment (ASE) ${ }^{58}$ interface, was used for the density functional theory (DFT) calculations. The exchange and correlation energy of the electrons was expressed within the concept of the generalized gradient approximation (GGA) by implementing the $\mathrm{RPBE}^{59}$ functional. The grid spacing was selected to be $\mathrm{h}=20 \AA$ while the atomic positions were relaxed until the total forces were lower than $0.05 \mathrm{eV} / \AA$. A $4 \times 4 \times 1$ Monkhorst-Pack ${ }^{60}$ k-point sampling for the $1 \times 3$ replicated $\mathrm{TiO}_{2}$ rutile (110) surface was used, with the last two layers (out of four) of the supper cell being constrained. The cubic configuration of the perovskite unit cell was used as the initial structure for the $\mathrm{SrTiO}_{3}$. A $10 \times 10 \times 10$ k-point mesh with a plane wave energy cut-off of $900 \mathrm{eV}$ and the RPBE functional were used to optimize the bulk structures. For optimizing the (100) $\mathrm{SrTiO}_{3}$ surface the same parameters as in the case of the rutile were used for a direct comparison between the two structures. 


\section{Experimental details}

All materials were prepared by spray-freeze freeze-dry synthesis. A total metal concentration of $8 \mathrm{mM}$ was maintained in all experiments. Titanium(IV) bis(ammonium lactato)dihydroxide (TBALD) solution (50 wt.\% in $\mathrm{H}_{2} \mathrm{O}$, Sigma Aldrich) was used as the source of titanium. $40 \mathrm{mM}$ concentrated stock solutions of TBALD in Milli-Q quality deionized water were prepared. As the source of transition metal dopants, vanadium(V) oxide (>98\%), chromium(III) nitrate nonahydrate (99\%), manganese(II) acetate tetrahydrate (>99\%), cobalt(II) acetate tetrahydrate (>98\%), and iron(II) acetate (>95\%) were all purchased from Sigma Aldrich and used as received. The precursor solutions for each experiment were prepared by dissolving the corresponding metal salts in Milli-Q quality deionized water and adding the respective amount of TBALD stock solution as the titanium source. The ratio of the initial metal precursors was adjusted to achieve the desired nominal composition; a total amount of 20 at.\% dopants with respect to titanium. For co-doping experiments, the dopants were added in a 1:1 ratio. The solutions were stirred for 30 minutes before the spray-freezing step. The ice precursors were prepared by spraying $100 \mathrm{~mL}$ of precursor solution into ca. $2 \mathrm{~L}$ of liquid nitrogen. The obtained ice precursor was freeze-dried using a FreeZone Triad Freeze Dry System 7400030 (Labconco) at reduced pressure ( $1 \mathrm{~Pa}$ ) according to the following temperature protocol: the temperature was kept constant at $-30{ }^{\circ} \mathrm{C}$ while the cooling chamber was evacuated, followed by a gradual increase of the temperature $\left[-30{ }^{\circ} \mathrm{C}(2 \mathrm{~h}),-25^{\circ} \mathrm{C}(5 \mathrm{~h}),-20^{\circ} \mathrm{C}(4 \mathrm{~h}),-15^{\circ} \mathrm{C}(6 \mathrm{~h})\right.$, $\left.+30^{\circ} \mathrm{C}(4 \mathrm{~h})\right]$. The obtained foam-like precursor was carefully removed from the freeze-dryer and annealed at $500{ }^{\circ} \mathrm{C}$ for $2 \mathrm{~h}$ in a muffle furnace.

The crystallinity and phase purity of the synthesized materials were analyzed by powder X-ray diffraction (XRD). The diffraction patterns were recorded using a Rigaku Miniflex 600 powder X-ray diffractometer with $\mathrm{Cu} \mathrm{K}_{\alpha}$ radiation operating at $30 \mathrm{kV}$ and $10 \mathrm{~mA}$. Rietveld refinements were performed to determine the unit cell parameter as well as contamination content with the Profex 3.13 .0 software package ${ }^{61}$ based on the BGMN Rietveld program. ${ }^{62}$

The morphology and particle size of all prepared samples were analyzed by scanning electron microscopy (SEM) using a Hitachi 54800 scanning electron microscope equipped with a Nanotrace EDX detector (Thermo Electron). The average sample composition was determined by energy-dispersive X-ray spectroscopy (EDX) measured at an accelerating voltage of $25 \mathrm{keV}$. The bandgap of the synthesized samples was determined by UV-Vis diffuse reflectance spectroscopy (DRS) (PerkinElmer LAMBDA 950).

The oxygen evolution activity of the prepared materials was assessed for all materials. The $\mathrm{TiO}_{2}$ electrodes were prepared by drop-casting on fluorine-doped tin oxide (FTO) 
glass (TEC 15, Dyesol, $15 \Omega / \mathrm{sq}$ ). The catalysts suspensions were prepared by dispersing $10 \mathrm{mg}$ of catalyst in a solution of $1 \mathrm{~mL} \mathrm{H} \mathrm{H}_{2} \mathrm{O}, 4 \mathrm{~mL}$ iPrOH, and $20 \mu \mathrm{L} 5 \%$ Nafion 117 solution (Sigma Aldrich). The electrode layer was deposited by dropping $10 \mu \mathrm{l}$ increments of the catalyst suspension onto the FTO substrate until a total catalyst loading of $100 \mu \mathrm{g} \mathrm{cm}^{-2}$ was reached. The deposited catalyst layer was dried in between each drop-casting step. The electrochemical experiments were performed in a threeelectrode set up using the respective doped $\mathrm{TiO}_{2} /$ FTO working electrode in combination with a platinum mesh as the counter electrode and a saturated calomel electrode (SCE) as the reference electrode. For potential control, an AUTOLAB (PGSTAT 30) potentiostat was used in all experiments. Voltammograms were recorded at a scan rate of $5 \mathrm{mV} / \mathrm{s}$ in $0.1 \mathrm{M} \mathrm{HClO}_{4}$ in a potential range of 1.3 to $2.0 \mathrm{~V}$ vs. $\mathrm{RHE}$, after cycling at $50 \mathrm{mV} / \mathrm{s}$ to achieve a steady surface capacitance.

All potentials were recalculated and are reported in the reversible hydrogen electrode (RHE) scale. Electrochemical impedance spectroscopy measurements were recorded in the range from $15 \mathrm{kHz}$ to $1 \mathrm{~Hz}$ with an amplitude of $10 \mathrm{mV}$ to estimate the ohmic drop of the solution. All voltammetric curves were corrected for uncompensated solution resistance. The reported current densities are based on the geometric surface area of the electrodes used. All electrochemical measurements were repeated at least three times to ensure reproducibility.

\section{Author contributions:}

T. Kutlusoy - Conceptualization, Methodology, Investigation, Visualization, Writing - original draft

S. Divanis - Conceptualization, Methodology, Investigation, Visualization, Writing - original draft, Writing - review \& editing

R. Marina - Investigation, Visualization Writing - original draft

R. Pittkowski - Methodology, Investigation, Visualization, Writing - review \& editing,

Supervision

P. Krtil - Writing - review \& editing, Supervision, Resources, Project administration

J. Rossmeisl - Conceptualization, Writing - review \& editing, Supervision, Resources, Project administration

\section{Conflict of interest}

There are no conflicts to declare.

\section{Acknowledgments:}

TK, SD, RM, and RP gratefully acknowledge the support from the EC Horizon 2020, Marie Sklodowska-Curie Actions (Contract No. 722614). The project was further supported by the Czech Science Foundation within project No. 21-03037S. We acknowledge support from the Danish National Research Foundation via grant DNRF-149. 


\section{References}

1. H. Ibrahim, A. Ilinca, and J. Perron, Renew. Sustain. Energy Rev. , 2008, 12, 1221.

2. M. Carmo, D. L. Fritz, J. Mergel, and D. Stolten, Int. J. Hydrog. Energy, 2013, 38, 4901.

3. J. Suntivich, K. J. May, H. a Gasteiger, J. B. Goodenough, and Y. Shao-Horn, Science, 2011, 334, 1383.

4. A. Grimaud, K. J. May, C. E. Carlton, Y.-L. Lee, M. Risch, W. T. Hong, J. Zhou, and Y. ShaoHorn, Nat. Commun., 2013, 4, 2439.

5. R. Frydendal, E. A. Paoli, I. Chorkendorff, J. Rossmeisl, and I. E. L. Stephens, Adv. Energy Mater., 2015, 5, 1500991.

6. C. C. L. McCrory, S. Jung, J. C. Peters, and T. F. Jaramillo, J. Am. Chem. Soc., 2013, 135, 16977.

7. Y. Lee, J. Suntivich, K. J. May, E. E. Perry, and Y. Shao-Horn, J. Phys. Chem. Lett., 2012, 3, 399.

8. M. Schalenbach, G. Tjarks, M. Carmo, W. Lueke, M. Mueller, and D. Stolten, J. Electrochem. Soc., 2016, 163, F3197.

9. G. Matute, J. M. Yusta, and L. C. Correas, Int. J. Hydrog. Energy, 2019, 44, 17431.

10. K. E. Ayers, E. B. Anderson, C. Capuano, B. Carter, L. Dalton, G. Hanlon, J. Manco, and M. Niedzwiecki, ECS Trans., 2019, 33, 3.

11. K. A. Stoerzinger, L. Qiao, M. D. Biegalski, and Y. Shao-Horn, J. Phys. Chem. Lett., 2014, 5, 1636.

12. F.-W. Wellmer, P. Buchholz, J. Gutzmer, C. Hagelüken, P. Herzig, R. Littke, and R. K. Thauer, "Raw Materials for Future Energy Supply", 2019, Springer International, Cham, Switzerland.

13. M. Bernt, A. Siebel, and H. A. Gasteiger, J. Electrochem. Soc., 2018, 165, F305.

14. R. K. Pittkowski, D. F. Abbott, R. Nebel, S. Divanis, E. Fabbri, I. E. Castelli, T. J. Schmidt, J. Rossmeisl, and P. Krtil, Electrochimica Acta, 2021, 366, 137327.

15. A. Grimaud, A. Demortière, M. Saubanère, W. Dachraoui, M. Duchamp, M.-L. Doublet, and J.-M. Tarascon, Nat. Energy, 2016, 2, 16189.

16. J. K. Nørskov, J. Rossmeisl, A. Logadottir, L. Lindqvist, J. R. Kitchin, T. Bligaard, and H. Jónsson, J. Phys. Chem. B, 2004, 108, 17886.

17. J. Rossmeisl, Z.-W. Qu, H. Zhu, G.-J. Kroes, and J. K. Nørskov, J Electroanal Chem, 2007, $607,83$.

18. I. C. Man, H.-Y. Y. Su, F. Calle-Vallejo, H. A. Hansen, J. I. J. I. Martínez, N. G. Inoglu, J. Kitchin, T. F. Jaramillo, J. K. Nørskov, and J. Rossmeisl, ChemCatChem, 2011, 3, 1159.

19. S. Divanis, T. Kutlusoy, I. M. Ingmer Boye, I. C. Man, and J. Rossmeisl, Chem Sci, 2020, 11, 2943.

20. L. G. V. Briquet, M. Sarwar, J. Mugo, G. Jones, and F. Calle-Vallejo, ChemCatChem, 2017, 9, 1261.

21. D.-Y. Kuo, H. Paik, J. Kloppenburg, B. Faeth, K. M. Shen, D. G. Schlom, G. Hautier, and J. Suntivich, J. Am. Chem. Soc., 2018, 140, 17597.

22. D.-Y. Kuo, J. K. Kawasaki, J. N. Nelson, J. Kloppenburg, G. Hautier, K. M. Shen, D. G. Schlom, and J. Suntivich, J. Am. Chem. Soc., 2017, 139, 3473.

23. R. K. B. Karlsson, A. Cornell, and L. G. M. Pettersson, Electrochimica Acta, 2015, 180, 514.

24. M. García-Mota, A. Vojvodic, H. Metiu, I. C. Man, H.-Y. Su, J. Rossmeisl, and J. K. Nørskov, ChemCatChem, 2011, 3, 1607.

25. A. FUJISHIMA and K. HONDA, Nature, 1972, 238, 37.

26. Á. Valdés, Z.-W. Qu, G.-J. Kroes, J. Rossmeisl, and J. K. Nørskov, J. Phys. Chem. C, 2008, $112,9872$.

27. N. Roy, Y. Sohn, K. T. Leung, and D. Pradhan, J. Phys. Chem. C, 2014, 118, 29499. 
28. D. M. Jang, I. H. Kwak, E. L. Kwon, C. S. Jung, H. S. Im, K. Park, and J. Park, J. Phys. Chem. C, 2015, 119, 1921.

29. T. Umebayashi, T. Yamaki, H. Itoh, and K. Asai, J. Phys. Chem. Solids, 2002, 63, 1909.

30. L. C. Seitz, D. Nordlund, A. Gallo, and T. F. Jaramillo, Electrochimica Acta, 2016, 193, 240.

31. M. García-Mota, A. Vojvodic, F. Abild-Pedersen, and J. K. Nørskov, J. Phys. Chem. C, 2013, 117, 460.

32. Y. Wang, R. Zhang, J. Li, L. Li, and S. Lin, Nanoscale Res. Lett., 2014, 9, 46.

33. Z. Yuan, J. Jia, and L. Zhang, Mater. Chem. Phys., 2002, 73, 323.

34. S. S. Srinivasan, J. Wade, E. K. Stefanakos, and Y. Goswami, J. Alloys Compd., 2006, 424, 322.

35. H. Luo, T. Takata, Y. Lee, J. Zhao, K. Domen, and Yan, Chem. Mater., 2004, 16, 846.

36. I. E. Castelli, I.-C. Man, S.-G. Soriga, V. Parvulescu, N. B. Halck, and J. Rossmeisl, J. Phys. Chem. C, 2017, 121, 18608.

37. X. Huang, J. Wang, H. B. Tao, H. Tian, and H. Xu, Chem. Sci., 2019, 10, 3340.

38. A. R. Akbashev, L. Zhang, J. T. Mefford, J. Park, B. Butz, H. Luftman, W. C. Chueh, and A. Vojvodic, Energy Env. Sci, 2018, 11, 1762.

39. M. Cui, T. Liu, Q. Li, J. Yang, and Y. Jia, ACS Sustain. Chem. Eng., 2019, 7, 15346.

40. J. P. Perdew, Int. J. Quantum Chem., 1985, 28, 497.

41. V. Tripkovic, H. A. Hansen, J. M. Garcia-Lastra, and T. Vegge, J. Phys. Chem. C, 2018, 122, 1135.

42. M. S. Whittingham, Curr. Opin. Solid State Mater. Sci., 1996, 1, 227.

43. J. Gopalakrishnan, Chem. Mater., 1995, 7, 1265.

44. N. Satoh, T. Nakashima, and K. Yamamoto, Sci. Rep., 2013, 3, 1959.

45. D. P. Macwan, P. N. Dave, and S. Chaturvedi, J. Mater. Sci., 2011, 46, 3669.

46. L. Kavan, M. Grätzel, S. E. Gilbert, C. Klemenz, and H. J. Scheel, J. Am. Chem. Soc., 1996, $118,6716$.

47. D. O. Scanlon, C. W. Dunnill, J. Buckeridge, S. A. Shevlin, A. J. Logsdail, S. M. Woodley, C. R. A. Catlow, Michael. J. Powell, R. G. Palgrave, I. P. Parkin, G. W. Watson, T. W. Keal, P. Sherwood, A. Walsh, and A. A. Sokol, Nat. Mater., 2013, 12, 798.

48. V. Petrykin, K. Macounova, O. A. Shlyakhtin, and P. Krtil, Angew. Chem. - Int. Ed., 2010, 49, 4813.

49. K. Minhová Macounová, M. Klusáčková, R. Nebel, M. Zukalova, M. Klementova, I. E. Castelli, M. D. Spo, J. Rossmeisl, L. Kavan, and P. Krtil, J. Phys. Chem. C, 2017, 121, 6024.

50. M. Klusáčková, R. Nebel, K. Minhová Macounová, M. Klementová, and P. Krtil, Electrochimica Acta, 2019, 297, 215.

51. M. Huynh, D. K. Bediako, and D. G. Nocera, J. Am. Chem. Soc., 2014, 136, 6002.

52. V. Tripkovic, H. A. Hansen, and T. Vegge, ChemSusChem, 2018, 11, 629.

53. E. B. Castro, C. A. Gervasi, and J. R. Vilche, J. Appl. Electrochem., 1998, $28,835$.

54. A. Moysiadou, S. Lee, C.-S. Hsu, H. M. Chen, and X. Hu, J. Am. Chem. Soc., 2020, 142, 11901.

55. M. Bajdich, M. García-Mota, A. Vojvodic, J. K. Nørskov, and A. T. Bell, J. Am. Chem. Soc., 2013, 135, 13521.

56. J. J. Mortensen, L. B. Hansen, and K. W. Jacobsen, Phys Rev B, 2005, 71, 35109.

57. J. Enkovaara, C. Rostgaard, J. J. Mortensen, J. Chen, M. Dułak, L. Ferrighi, J. Gavnholt, C. Glinsvad, V. Haikola, H. A. Hansen, H. H. Kristoffersen, M. Kuisma, A. H. Larsen, L. Lehtovaara, M. Ljungberg, O. Lopez-Acevedo, P. G. Moses, J. Ojanen, T. Olsen, V. Petzold, N. A. Romero, J. Stausholm-Møller, M. Strange, G. A. Tritsaris, M. Vanin, M. Walter, B. Hammer, H. Häkkinen, G. K. H. Madsen, R. M. Nieminen, J. K. Nørskov, M. Puska, T. T. Rantala, J. Schiøtz, K. S. Thygesen, and K. W. Jacobsen, J. Phys. Condens. Matter, 2010, 22, 253202. 
58. A. Hjorth Larsen, J. Jørgen Mortensen, J. Blomqvist, I. E. Castelli, R. Christensen, M. Dułak, J. Friis, M. N. Groves, B. Hammer, C. Hargus, E. D. Hermes, P. C. Jennings, P. Bjerre Jensen, J. Kermode, J. R. Kitchin, E. Leonhard Kolsbjerg, J. Kubal, K. Kaasbjerg, S.

Lysgaard, J. Bergmann Maronsson, T. Maxson, T. Olsen, L. Pastewka, A. Peterson, C. Rostgaard, J. Schiøtz, O. Schütt, M. Strange, K. S. Thygesen, T. Vegge, L. Vilhelmsen, M. Walter, Z. Zeng, and K. W. Jacobsen, J. Phys. Condens. Matter, 2017, 29, 273002.

59. B. Hammer, L. B. Hansen, and J. K. Nørskov, Phys Rev B, 1999, 59, 7413.

60. H. J. Monkhorst and J. D. Pack, Phys Rev B, 1976, 13, 5188.

61. N. Doebelin and R. Kleeberg, J. Appl. Crystallogr., 2015, 48, 1573.

62. J. Bergmann, P. Friedel, and R. Kleeberg, CPD Newslett Commiss Powder Diffract Int Union Crystallogr, 1998, 20, 5. 